\title{
Development of a speech controlled water tap and fan system using linear predictive coefficient for feature extraction
}

\author{
Nnamdi S. Okomba ${ }^{1 *}$, Adebimpe O. Esan ${ }^{1}$, Bolaji Omodunbi ${ }^{1}$, Ibrahim A. Adeyanju ${ }^{1}$, Sulaimon A. Bashir ${ }^{2}$ \\ ${ }^{1}$ Department of Computer Engineering, Federal University Oye-Ekiti, Ekiti State, Nigeria \\ ${ }^{2}$ Department of Computer Science, Federal University of Technology Minna, Nigeria \\ *Corresponding author E-mail: nnamdi.okomba@fuoye.edu.ng
}

\begin{abstract}
Automatic device control is of great assistance in different electronic application areas that will aid human endeavors. Traditionally, fan and water pump system used in many homes are controlled by using manual process. This is a time consuming and difficult procedure, for the elderly, and handicap persons. In this view, this paper presents a microcontroller based fan and water pump control system using speech recognition. The system has a PIC16F628A microcontroller activated by a speech recognizer, then triggers control to a DC motor which set the water tap or the fan in motion. The Spoken words chosen for recognition are ON and OFF and the speech samples were extracted with Linear Predictive Coefficient (LPC) Feature extraction technique and after experimenting with the developed system, the result indicates the device is of good performance. We intend to increase the complexity of this work by adding specific sensors that will automatically switch off the fan at specified room temperature, and turn off the water tap at the fill of a container.
\end{abstract}

Keywords: Speech Recognition; Linear Predictive Coefficient; Microcontroller; Features Extraction.

\section{Introduction}

The use of speech recognition technology in automation control greatly improves the usability and functionality of any modern home [1]. Automatic control using speech recognition technology processes the speech signal received and execute the command. The process is highly beneficial because it provides a natural and intuitive way of controlling household appliances using speech instead of the hands.

Linear predictive coefficient (LPC) is a feature extraction technique that reduces the bitrates of the speech signal, thereby reducing the size of the transmitting signal, and thus requires less bandwidth. LPC algorithm uses the encryption of data so the data is secured until the destination [2].

Automation involves device control with the aid of sensors, microcontrollers, and actuators with minimal or no human interference [3]. This advanced technology is used to do automation of house activities [4].

Switching of home appliances using speech recognition technique has lower power consumption [5]. It is mainly focused on the elderly people, disables and for the people who are unable to type text or face difficulties in typing. For the disable people, it is quite difficult to operate the home appliances physically or they are unable or feel uncomfortable to type a text so as to switch on/off the relative device as in [6].

\section{Related works}

An intelligent home navigation system (IHNS) is proposed to facilitate the elderly and the physically challenged persons [7]. It proposes an idea of an automated voice based home navigation system. The system comprises over a wheelchair, navigation module and speech recognition module SR-07 and a line follower module for navigation. The system has predefined voice commands relating to different rooms, and predefined routes relating to those rooms for the navigation. There is also a collision avoidance system installed in the project.

The intelligent access control system is developed that is based on SPCE061A voice recognition chip [8]. The supporting software comprises of the voice training module, the voice recognition module, the voice data processing module and the voice-playing module. The system completes the functions of collecting the voice data, distilling character, special voice recognition and voice playing in terms of initializing the system and the identification training.

The proposed system in [9] comprises over a voice controlled wheel chair. The system is built using low cost speech recognition board and a microcontroller. The speech recognition board utilizing HM2007 speech recognition chip. It uses an idea of paired-word for issuing a command to the system, so that if words similar to the voice commands are spoken within the vicinity of the voice recognition kit, they are not accidently detected. The system is speaker dependent and also an isolated speech system, to avoid further accidental voice detections. 
Voice commands are used in [10] to switch the lights ON/OFF and regulating the speed of appliances. Using AVR (Advanced Virtual RISC) studio, to run program required for proposed system work, while the Practical speech recognition kit is utilized in order to store and recognize the user's voice and achieving a recognition accuracy of more than $90 \%$. Moreover, this project also helps in efficient use of the electricity which is an important constraint in day to day life.

\section{Methodology}

There are several steps and operations applied in designing microcontroller based speech recognition system for controlling home fan and water pump. The block diagram of the system is specified in figure 1 and has six relevant stages which include data acquisition, preprocessing, feature extraction, recognition, actuator control, and LCD display.

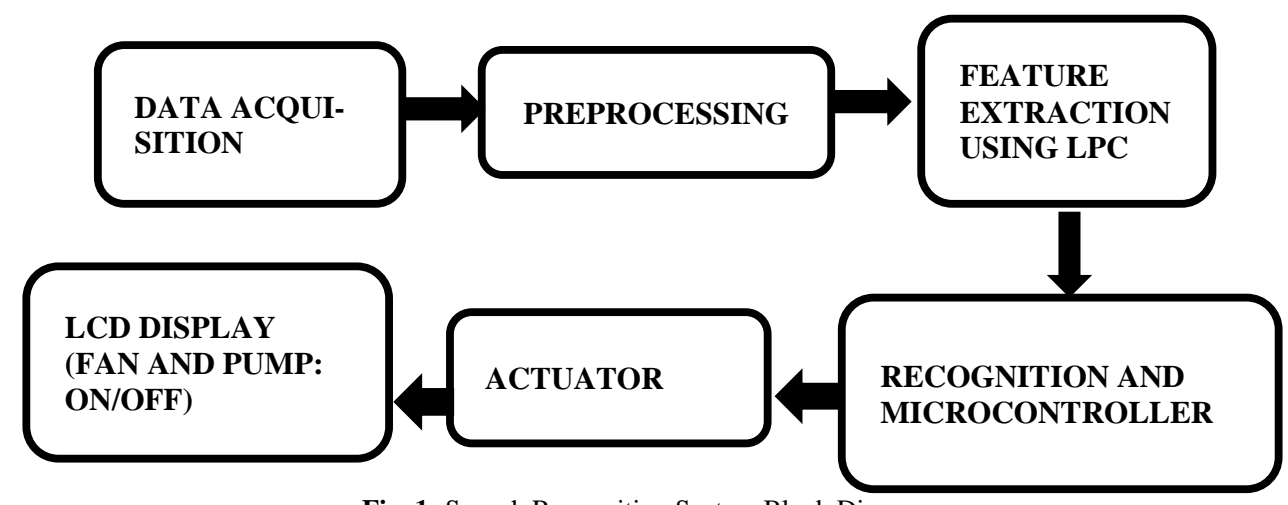

Fig. 1: Speech Recognition System Block Diagram.

The speech recognition aspect of this design is built using MATLAB software with series of operations that resulted to recognition of the speech of a user. The data acquisition process involves capturing speech samples with a computer system microphone under MATLAB environment and representing them in appropriate notations, after which the preprocessing stage purifies the acquired speech signals, then the creating of feature vectors to represent time-slices of the converted input speech signal using linear prediction coefficient feature extraction follows. Matching the results against a library of known speech waveforms and selecting the most likely sequence of words is then performed at the speech recognition stage. The PIC16F628A microcontroller receives signal from the speech recognition system developed in MATLAB and controls the fan and water pump model operations. These are the mathematical tools and algorithm used while processing the user(s) speech signal namely; Linear Predictive Coefficient (LPC) algorithm for feature extraction and vector quantization using the Linde-Buzo-Gray algorithm (VQLBG) for easy recognition of user speech signals. Figure 2 illustrate the operational flow diagram of the design.

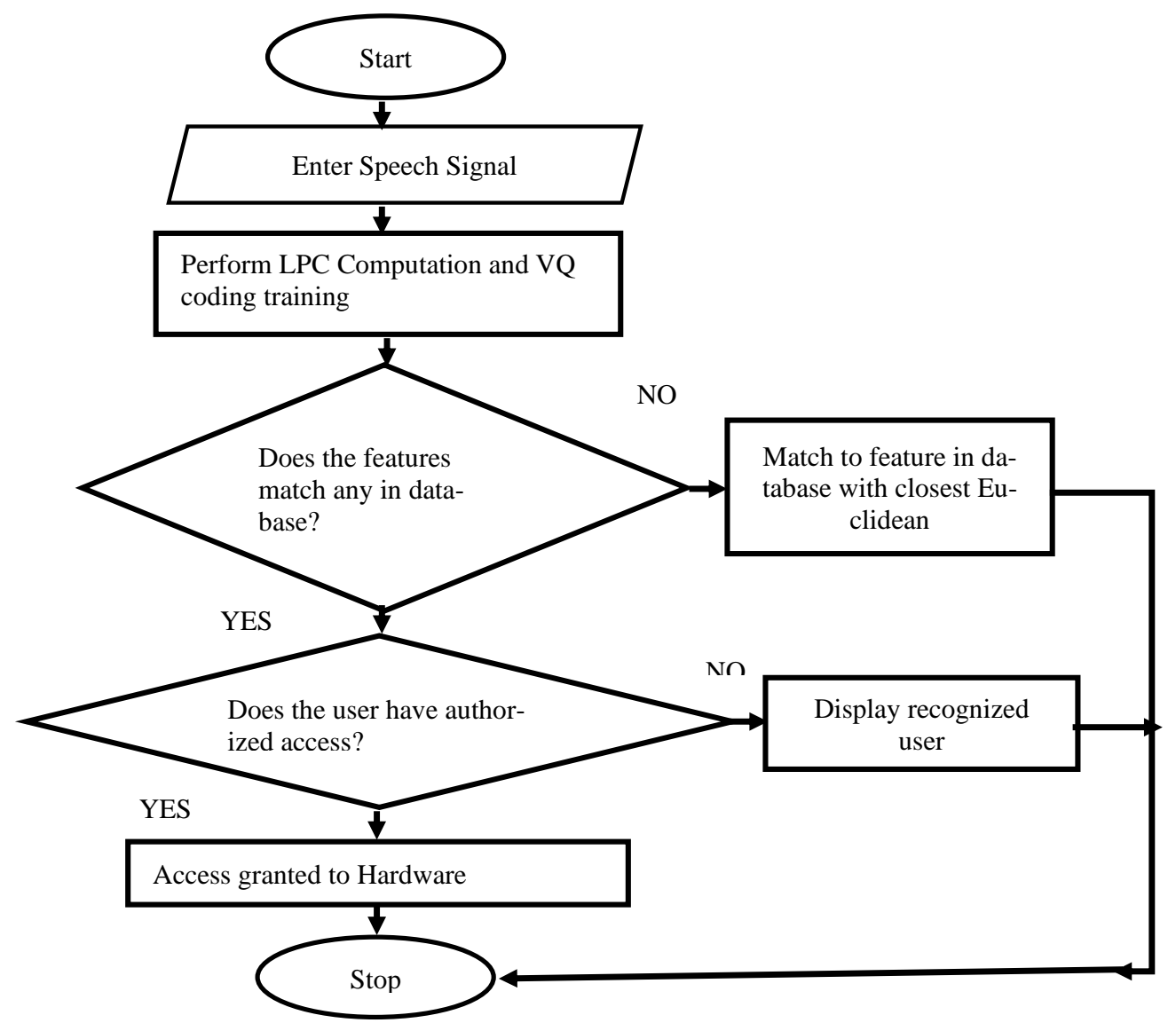

Fig. 2: Operational Flow Diagram of the Speech Controlled System. 


\section{Implementation}

\subsection{Data acquisition}

The acquisition stage of the speech recognition system, requires some parameters to be input for pre-processing of the user speech. The parameters are represented as a class number or sound ID for recognizing individual speech in the database; these parameters are the sampling frequency $(22050 \mathrm{~Hz})$; Sampling bit (8); and the duration of recording ( 3 seconds). After the pre-processing, the feature vectors of the speech signals were extracted using linear predictive coefficient and stored in the system database for matching. Figure 3 illustrates the command Prompt and Pop-up Message Box display for the Speech Acquisition.

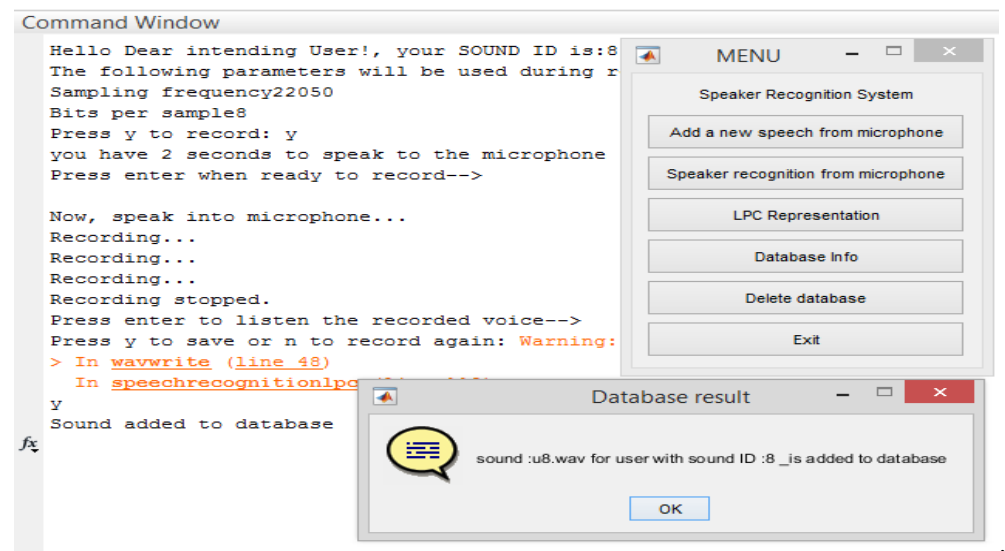

Fig. 3: Command Prompt and Pop-up Message Box Display for the Speech Acquisition.

\subsection{Recognition of speech features}

As shown in figure 4, six different speakers insert their speech data to the system through an analogue microphone. The system performs LPC coefficients computation to get the feature vectors whose cepstral representation is as shown in figure 5, after which VQ codebook training and matching based on the closeness of the parametric distance (Euclidean distance) is carried out. The access control allows only one authorize user to have access to the operation control of the hardware prototype. On recognition of the authorized user, a signal is sent from the MATLAB through the RS232 program cable to activate the hardware prototype.

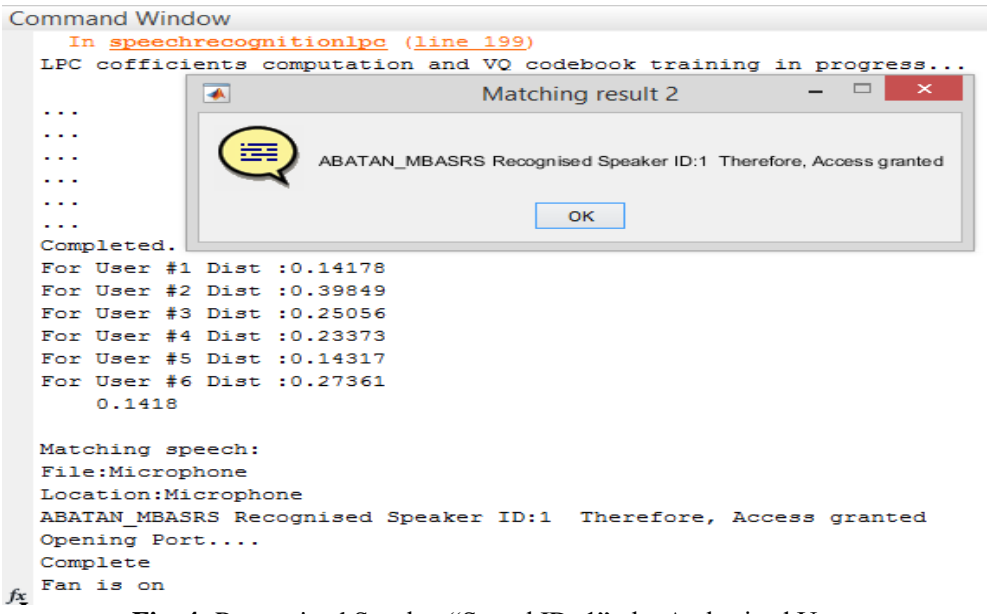

Fig. 4: Recognized Speaker "Sound ID: 1", the Authorized User.

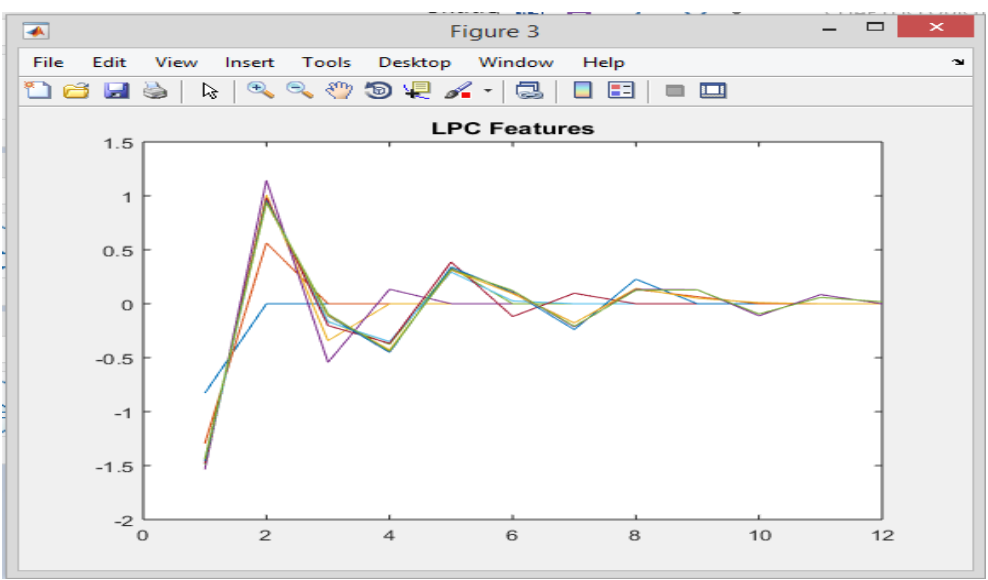

Fig. 5: Extracted LPC Cepstral Features of the Users Speech Sample. 


\subsection{Hardware implementation}

The PIC16F628A microcontroller receives access signal from MATLAB after the speech recognition through a COM port (RS232serial program cable) and sends a signal to trigger the water pump and fan controlled system while the LCD displays the status of the system. Figure 6 illustrates the schematic diagram of the water pump and fan controlled system. Pin 17 and Pin 18 of the PIC16F628A is connected to the $2 \mathrm{~N} 2222 \mathrm{~A}$ transistors which controls the DC motor to power on and off the submersible water pump for water flow and fan rotation respectively. Pin 10-13 of PIC16F628A is connected to pin 6-3 of the LCD connector which is equivalent to pin 11-14 on the LCD itself, it is used to send command or data to the LCD.

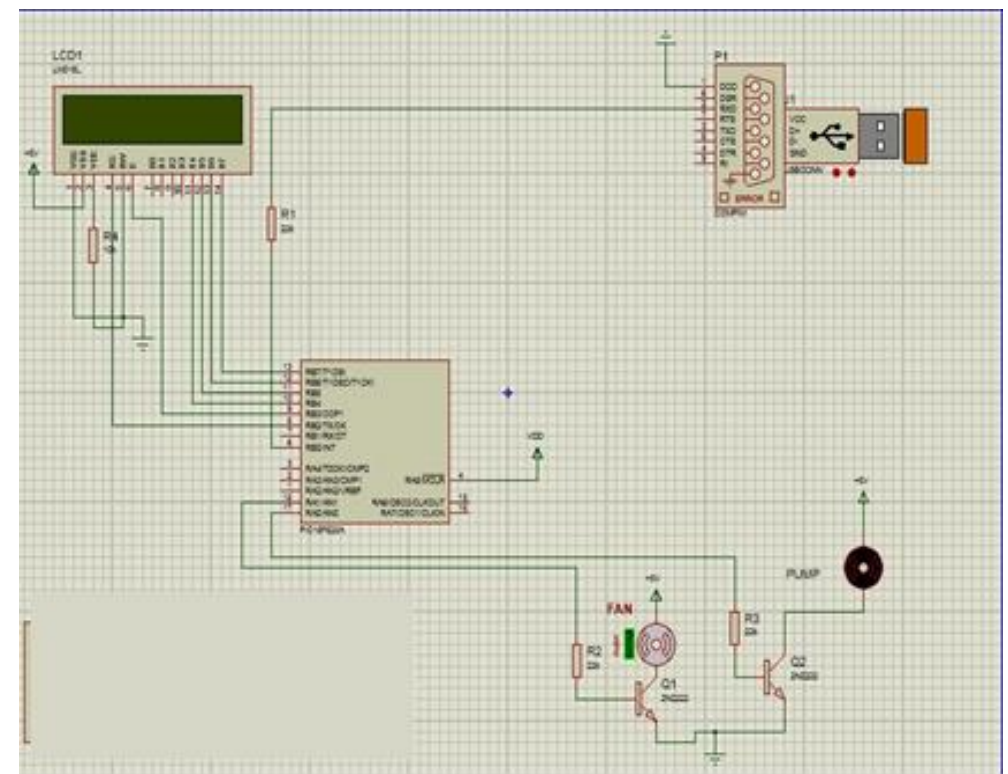

Fig. 6: Schematic Diagram of the Water Pump and Fan Controlled System.

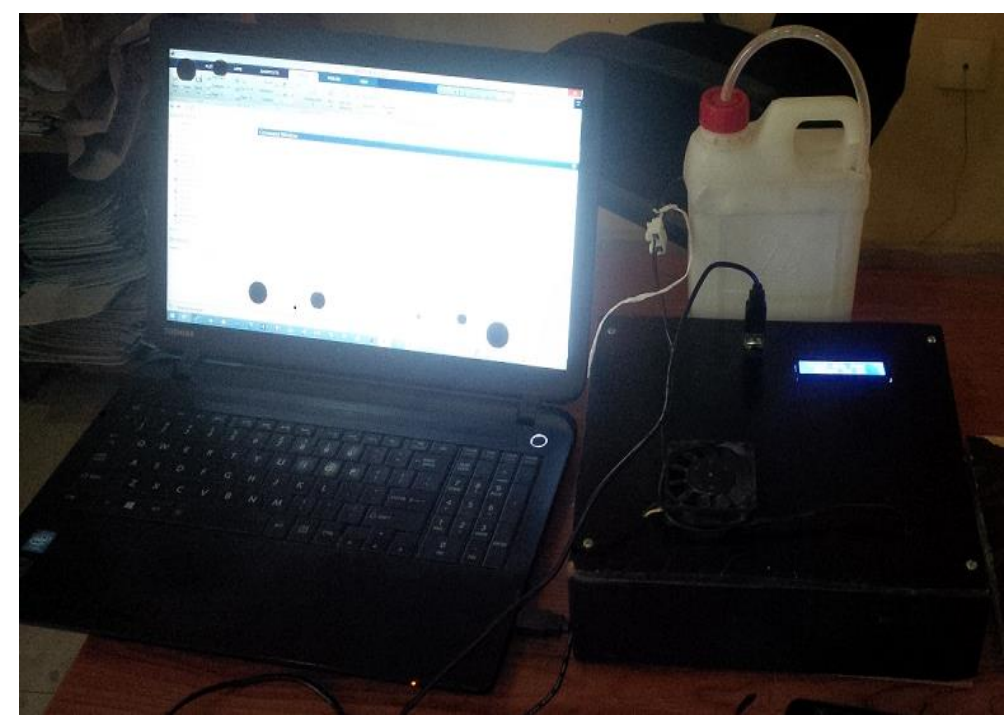

Fig. 6: The Prototype of Fan and Water Pump Control Using Speech Recognition.

\subsection{Parametric representation of recognition process of users}

Table 1: LPC coefficients of Speakers to Speech Samples BREEZE and WATER

\begin{tabular}{llll}
\multicolumn{4}{c}{ Table 1: LPC coefficients of Speakers to Speech Samples BREEZE and WATER } \\
\hline & No Of Trials & Speech Sample: [Breeze] & Speech Sample: [Water] \\
\hline \multirow{3}{*}{ Speaker 1 } & 1 & $7.2033-0.0266-0.6530$ & $7.04990 .0470-0.4099$ \\
& 2 & $6.88610 .0459-0.2512$ & $7.24850 .0654-0.4107$ \\
& 3 & $7.10460 .0356-0.4330$ & $7.87000 .2526-0.4656$ \\
& 4 & $6.6582-0.0457-0.5206$ & $6.51710 .1187-0.2738$ \\
& 5 & $7.31130 .0450-0.3245$ & $6.9053-0.0652-0.6109$ \\
Speaker 2 & 1 & $6.68020 .0440-0.4454$ & $6.6943-0.0174-0.3318$ \\
& 2 & $6.7932-0.0813-0.6058$ & $6.7742-0.0252-0.3276$ \\
& 3 & $6.78740 .0581-0.2692$ & $6.63320 .0482-0.3426$ \\
& 4 & $6.67460 .1455-0.2800$ & $6.86020 .0096-0.4060$ \\
\hline
\end{tabular}

Table 1 illustrates the LPC feature vectors parametric representation of the speech sample BREEZE and WATER collected from two speakers over a repeated trial of the data capture up to five times each. The variation in the feature vectors is due to distortion arising in the channel of data transmission either by noise or intonation. 
Table 2: Euclidean Distance of Speakers to Speech Samples BREEZE and WATER

\begin{tabular}{llll}
\hline $\begin{array}{l}\text { User in Data- } \\
\text { base }\end{array}$ & $\begin{array}{l}\text { Speakers to be recognized using the individual Euclidean distance of features extracted to the features of data stored in the speech } \\
\text { system database } \\
\text { Speech Sample: [ BREEZE ] } \\
\text { Distance to User-1 }\end{array}$ & Distance to User-2 & Distance to User-3 \\
\hline Speaker-1 & 0.27574 & 0.37012 & 0.72682 \\
Speaker -2 & 0.22788 & 0.38932 & 0.71056 \\
Speaker -3 & 0.21898 & 0.36737 & 0.60802 \\
Speech Sample: [ WATER ] & & 0.29976 \\
Speaker - 1 & 0.33991 & 0.34818 & 0.31292 \\
Speaker -2 & 0.28496 & 0.29728 & 0.53835 \\
Speaker -3 & 0.33461 & 0.30088 & 0.60194 \\
\hline
\end{tabular}

Table 2 above summarize the result and evaluate the system based on the Euclidean distance of the individual speech signal to be. Here, the parametric representation of the distances of the speech signal is shown as calculated when the LPC and VQ codebook training function are applied. The speaker having the shortest feature distance is matched to a user stored in the database of the speech system, and thus, is the recognized user. In the table above, the red sections simply indicate the recognized users.

\section{Conclusion}

This paper has discussed the development of speech controlled water pump and fan system as extracted speech features were used to activate the control of a fan or water pump. The major attribute of this design is the multitasking approach of the system, encompassing the speech recognition and the control system of fan, water tap, and display. An advantage of a speech controlled water pump and fan system is that it will boost the flexibility of accessing this appliance with comfort and ease. A noticeable limitation of the designed speech controlled water pump and fan system is the limited number of users the database can contain and the complex hardwired nature of the system. Irrespective of this setback, the designed system will still serve a great purpose in home automation assistance. Hopefully, more sophisticated mobile wireless speech controlled device with artificial intelligence will be a recommendation for future design.

\section{References}

[1] R. A. Aqeel-ur-Rehman, and H. Khursheed,. Voice Controlled Home Automation System for the Elderly or Disabled People. J. Appl. Environ. Biol. Sci, vol. 4, (2014) pp. 55-64.

[2] R. Jain, and S. K Saxena. Advanced Feature Extraction and Its Implementation in Speech Recognition System, International Journal of Services Technology and Management (IJSTM), Vol. 2 Issue 3. (2011).

[3] A. K Gupta, and S. K Arora. Industrial Automation and Robotics, 348 pages, Laxmi Publications, ISBN-10: 8131801810. (2007).

[4] J. Burroughs, X-10 Home Automation Using the PIC16F877A Lamp, vol. 10, (2010) article 10.

[5] S. M. AnamulHaque, S. M. Kamruzzaman, and M.D. Ashraful Islam1. A System for Smart-Home Control of Appliances Based on Timer and Speech Interaction, Proceedings of the 4th International Conference on Electrical Engineering and 2nd Annual Paper Meet 26-28, pp. 128-131, January, 2006.

[6] B. Mardiana, H. Hazura, S. Fauziyah, M. Zahariah, A.R. Hanim, and M.K. Noor Shahida,. Homes Appliances Controlled Using Speech Recognition in Wireless Network Environment, ICCTD, vol. 2, pp.285- 288, 2009 International Conference on Computer Technology and Development, (2009). https://doi.org/10.1109/ICCTD.2009.178.

[7] J. Gerhart. Home automation and wiring. Mcgraw-hill/TAB Electronics; 1 edition (March 31, 1999). (ISBN 0070246742) ISBN-13: 9780070246744. (1999).

[8] W. C. Mann, (Ed.) Hoboken. Smart technology for aging, disability, and independence: The state of the science. John Wiley \& Sons. Inc., 2005. 379 pages, (ISBN 0-471-69694-3). (2005).

[9] R. Nave,R. (2014). Hyperphysics Microphones. Retrieved2014, May, from http://hyperphysics.phyastr.gsu.edu/hbase/audio/mic.html

[10] W. Yuwraj, T., Ankita, G., Pallavi, and D. Ruchita, Voice Recognition System for Home Appliances, International Journal of Innovative Research in Electrical, Electronics, Instrumentation and Control Engineering Vol. 4, Issue 2, February 2016 Copyright to IJIREEICE DOI 10.17148/IJIREEICE.2016.4221 79. IJIREEICE ISSN (Online) $2321 \quad-\quad 2004$ ISSN $\quad$ (Print) $2321 \quad-\quad 5526 . \quad$ (2016). https://doi.org/10.17148/IJIREEICE.2016.4221. 